\title{
Le groupe de travail «Qualité» de la FMH à la recherche de nouveaux membres!
}

\author{
Martina Hersperger, \\ responsable du département \\ $D D Q$
}

Peltenburg M, Kernen H, Schneider P, von Below GC, Waldis $\mathrm{G}$, et al. La qualité: une interaction de toutes les forces en présence dans le domaine médical. Bull Méd Suisses. 2005; 86(20):1215-20.
Depuis 2005, le groupe de travail «Qualité» (GTQ) est une commission consultative du Comité central. Placé sous la direction d'Olivier Kappeler, membre du CC et responsable du domaine DDQ (données, démographie et qualité), il comprend un groupe de pilotage et une équipe d'experts. Le groupe de pilotage se compose de six à huit membres de la FMH (il comptait jusqu'à présent deux personnes de Suisse romande et quatre de Suisse alémanique) en activité professionnelle dans le secteur ambulatoire ou hospitalier. Quant à l'équipe d'experts, elle a pu gagner à sa cause un délégué d'une organisation internationale, un délégué des Hautes écoles et un délégué des assureurs. Le département DDQ quant à lui accomplit un travail de soutien et de coordination.

\section{Tâches du groupe de travail «Qualité»}

- Développer les lignes directrices «Qualité médicale»* et les mettre en œuvre

- Inciter à clarifier les concepts et à développer la qualité au sein du corps médical, puis à les appliquer

- Elaborer des bases visant à l'interaction des réglementations, des capacités et des résultats

- Préparer des prises de position à l'attention du Comité central de la FMH

- Entamer, sur la base de la conception de la qualité du corps médical suisse, des négociations avec les interlocuteurs concernés

Après la démission d'un délégué francophone, le groupe de travail «Qualité» cherche 1 à 2 membres pour son groupe de pilotage, de préférence de la Suisse romande ou du Tessin.

\section{Tâches du groupe de pilotage}

- Piloter l'ensemble du programme

- Garantir le professionnalisme et la qualité (avec l'équipe d'experts)

- Intégrer les tendances actuelles et les derniers développements

- Elaborer des prises de position sur les sujets traités et des faits d'actualité

- Assurer la gestion des groupes impliqués

- Collaborer occasionnellement à des projets

\section{Exigences, implication et indemnisation}

- Intérêts pour la qualité médicale et expérience de ce domaine

- Compréhension de l'allemand et du français

- Membre de la FMH

- 6 séances par année $(5 \times$ une demi-journée, $1 \times$ un jour entier), y c. préparation et suivi

- Les séances ont lieu à Berne (en règle générale)

- Indemnisation selon le Règlement concernant les frais du Secrétariat général de la FMH

- Indemnisation selon règlement particulier lors de collaboration à des projets

\section{Intéressé/e?}

Vous avez la possibilité de participer à une séance à titre d'invité/e. Les membres suivants du groupe de pilotage vous donneront volontiers des renseignements et se réjouissent que vous preniez contact avec eux:

Olivier Kappeler, membre du Comité central, Domaine DDQ: olivier.kappeler@hin.ch

Patrick Bovier, membre suisse romand, groupe de pilotage: patrick.bovier@hcuge.ch

Martina Hersperger, responsable du département DDQ: martina.hersperger@fmh.ch 